\title{
entropy
}

ISSN 1099-4300

www.mdpi.org/entropy/

\section{Simple Entropic Derivation of a Generalized Black-Scholes Option Pricing Model}

\section{Michael J. Stutzer}

Dept. of Finance, 108 PBAB, Univ. of Iowa, Iowa City, IA., USA

E-mail: michael-stutzer@uiowa.edu

Received: 19 January 2000 / Accepted: 24 March 2000 / Published: 4 April 2000

\begin{abstract}
A straightforward derivation of the celebrated Black-Scholes Option Pricing model is obtained by solution of a simple constrained minimization of relative entropy. The derivation leads to a natural generalization of it, which is consistent with some evidence from stock index option markets.
\end{abstract}

Keywords: Option Pricing, Entropic Martingale Measure, Black-Scholes, Asset Pricing.

(C) 2000 by the author. Reproduction is permitted for noncommercial purposes. 


\section{A Simple Black-Scholes Derivation}

Consider the following discrete time approximation of a stochastic process for an asset's rate of return over periods of length $\Delta t$ :

$$
\frac{\Delta p}{p}=\mu \Delta t+\sigma \sqrt{\Delta t} \Delta z
$$

In (1), the random shocks $\sqrt{\Delta t} \Delta z \sim N(0, \Delta t)$ occur independently at each time step, which in the continuous time limit $\Delta t \rightarrow 0$ produce the independent standard Weiner process increments of that continuous time process. In (1), the mean return is $\mu \Delta t$ and the return variance is $\sigma^{2} \Delta t$, and we commensurately assume that the riskless rate of return is $r \Delta t$.

Now consider the general problem of pricing a derivative security in a discrete time economy, whose payoff at some time $T$ (years) depends solely on the price of a single underlying asset, e.g. a European call option on a traded stock. In the absence of arbitrage opportunities constructed by frictionless trading of the underlying and riskless assets with complete markets, the price of any derivative security with payoff at time $T$ (years) will be the risklessly discounted, expected value of its payoff under an equivalent martingale measure [18]. When the underlying asset's return $\Delta p / p$ is IID, as in (1), the density of the martingale measure is the $[T / \Delta t]$-fold product of the conditional risk neutral density $d Q / d P$ at each trading time, where the integer $[T / \Delta t]$ is the number of trading periods to expiration and $P$ denotes the actual conditional return distribution. The conditional risk neutral density $d Q / d P$ must satisfy the so-called martingale restriction [22]: ${ }^{1}$

$$
r \Delta t-E\left[\frac{\Delta p}{p} \frac{d Q}{d P}\right]=0
$$

Now consider the relative entropy minimizing conditional risk neutral density solving the linear inverse problem with constraint (2), i.e.

$$
\arg \min _{\frac{d Q}{d P}} \int \log \frac{d Q}{d P} d Q \text { s.t. }(2) .
$$

The solution to the linear inverse problem (3) is the following Gibbs Canonical density:

$$
\frac{d Q}{d P}=\frac{e^{\hat{w} \frac{\Delta p}{p}}}{E\left[e^{\hat{w} \frac{\Delta p}{p}}\right]} .
$$

\footnotetext{
${ }^{1}$ The martingale restriction (2) may alternatively be written as the constraint that the risklessly discounted, conditional risk neutral expected payoff of the underlying asset at $s+\Delta t$ must be its current price.
} 
where

$$
\hat{w}=\arg \max r \Delta t w-\log E\left[e^{w \frac{\Delta p}{p}}\right]
$$

For proofs of this well-known solution (4) and (5), see Ben-tal [4, sec.3], Bucklew [6, pp.30-32] and Csiszar [8, sec.3a].

The following proposition applies (3) to the problem of pricing a call option on an asset with return process (1) with normally distributed shocks.

PROPOSITION: When asset returns are generated by the IID process (1) with normally distributed shocks $\Delta z \sqrt{\Delta t} \sim N(0, \Delta t)$, the martingale (i.e product) density formed from the conditional density (3) is that used to calculate the Black-Scholes option pricing formula.

\section{PROOF OF PROPOSITION:}

Substitute (1) into (5), and factor the (nonstochastic) drift term out of the expectation to obtain:

$$
\hat{w}=\arg \max w(r-\mu) \Delta t-\log E\left[e^{w \sigma \sqrt{\Delta t} \Delta z}\right]
$$

Because $\sigma \sqrt{\Delta t} \Delta z \sim N\left(0, \sigma^{2} \Delta t\right)$, the latter term in (6) is the log moment generating function of this normally distributed random variable. Substituting this quadratic function of $w$ into (6) yields

whose solution is:

$$
\hat{w}=\arg \max w(r-\mu) \Delta t-w^{2} \frac{\sigma^{2}}{2} \Delta t
$$

$$
\hat{w}=\frac{(r-\mu)}{\sigma^{2}}
$$

Substituting (7) and (1) into (4), note that the drift term cancels, yielding the relative entropy minimizing conditional risk neutral density:

$$
\frac{d Q}{d P}=\frac{e^{-\lambda \sqrt{\Delta t} \Delta z}}{E_{P}\left[e^{-\lambda \sqrt{\Delta t} \Delta z)}\right]}
$$

where $\lambda \equiv \frac{\mu-r}{\sigma}$, and the expectation is taken with respect to $P \sim N(0, \Delta t)$.

Now take the logarithm of the $[T / \Delta t]$-fold product of the density (8), and simplify to find:

$$
\log \prod_{s=1}^{[T / \Delta t]} \frac{d Q}{d P}=-\lambda W_{T}-\frac{1}{2} \lambda^{2} T
$$

where $W_{T} \sim N(0, T)$. In a lengthy derivation using martingale theory, Dothan [9, pp.210-214] shows that (9) is the log of the density of the equivalent martingale measure used to compute the Black-Scholes option pricing formula as a risklessly discounted expected present value of a call option's payoff. 


\subsection{Entropic Option Pricing Literature Review}

There are many other uses of entropy in finance and economics. Because this is an option pricing paper, this review will focus on entropic reasoning applied to option pricing - a literature that has developed in small pockets within finance, actuarial, operations research, mathematics, and statistics departments that are largely isolated from one another. From my perspective as a finance professor, the novelty of the above derivation lies in its straightforward use of the process probabilities from (1), with normally distributed shocks, to compute the Black-Scholes martingale measure as the product of the relative entropy minimizing conditional risk-neutral probabilities. A 1994 publication of mine (Stutzer [26]) used Hua He's [20] incomplete market, discrete multinomial representation of the lognormal stock price process, instead of (1). As a result, the Black-Scholes martingale measure could only be derived as a continuous time limit of the discrete-time product measure. That paper reviewed earlier uses of entropy in finance and economics, noting that Cozzolino and Zahner [7] derived the lognormal stock price distribution itself, as that maximizing Shannon entropy among distributions with prescribed mean and variance. Using this reasoning as part of his Ph.D dissertation, Gulko [16] prescribed the riskfree mean and the actual variance to derive the risk-neutral lognormal price distribution used by many, e.g. Stoll and Whaley [25, Chap.11], to derive the Black-Scholes formula as a risklessly discounted expected value of the option's payoff at expiration. A recent publication by Fritelli [14] derives the relative entropy minimizing martingale measure under incomplete markets ${ }^{2}$, and demonstrates the connection between it and maximization of exponential utility. This connection was also noted by Samperi [24]. In the related context of arbitrage-free, discrete time asset pricing model diagnostics, the exponential utility connection was established earlier by Stutzer [27, sec.3.2.3].

Perhaps the best entropic derivations of other option pricing formulae are presented in Gerber and Shiu [15] and in Heston [21], which applies a somewhat broader class of risk-neutral transformations to alternative price processes. A price process-free, entropic method of option pricing is presented in Stutzer [28], applied by Zou and Derman [29], and extended in Foster and Whiteman [13].

All of these papers predict option prices without use of other observed market option prices. But a related "inverse" problem uses relative entropy minimization (or maximization of Shannon entropy) to derive a risk-neutral distribution that is constrained to be consistent with a subset of observed option prices. Arnold Zellner pointed out to me that perhaps the earliest paper on this inverse problem was Massimb [23], but the idea was developed further by Buchen and Kelly [5], and also by Hawkins, Rubinstein, and Daniell [19]. Later generalizations of the idea are seen in Avellanada [2], who constrains the measure to be consistent with observed market forward rate

\footnotetext{
${ }^{2}$ Stutzer [26] noted the earlier term "minimal" martingale measure of Follmer and Schweizer [12]
} 
agreements and interest rate swaps.

\section{Non-Normality: Reinterpreting the Black Scholes Volatility Parameter}

The Black-Scholes option pricing formula does not work well when the underlying asset is an index portfolio of stocks. Bates [3, p.25] reports that upon inverting the Black-Scholes call option pricing formula $B S(\sigma)^{3}$ to find the different number $\sigma_{i m p}$ that makes the Black Scholes formula value equal to each observed option market price, such implied volatilities are "upwardly biased relative to realized volatility...". This upward bias in stock index volatility was also noticed in a systematic study of $S \& P 100$ index option prices by Fleming [11], whose results are summarized later as providing empirical evidence for the following model.

Because this bias is indicative of non-normally distributed returns, we adopt the entropic hypothesis (4) and (5) with a possibly non-normally distributed $\sqrt{\Delta t} \Delta z$ in the return process (1). In order to retain the simplicity and familiarity of the Black-Scholes formula, use a time series of asset returns to nonparametrically estimate the solution $\hat{w}$ to $(5)$ and then use the standard deviation of the resulting estimated density (4) to compute a parameter, called $\hat{\sigma}$, that will replace the realized asset volatility $\sigma$ in the Black Scholes formula. $B S(\hat{\sigma})$ can be computed and compared to both the uncorrected Black Scholes value $B S(\sigma)$ and the market price $B S\left(\sigma_{\text {imp }}\right)$. The result will thus be a reinterpretation of the volatility parameter in the Black Scholes formula, needed to help correct for non-normalities. ${ }^{4}$

Specifically, obtain a time series sample of $\Delta t$-returns $\Delta p / p$, and substitute the sample average corresponding to the expectation operator used to define (5). Then, use the Newton-Raphson method to numerically maximize the resulting sample counterpart of (5), producing an estimate $\tilde{w}$ of $\hat{w}$. Under mild regularity conditions, Amemiya [1] shows that the extremum estimator $\tilde{w}$ is a consistent estimator of $\hat{w}$. Substitute $\tilde{w}$ into the density (4), divide this density's variance $\hat{\sigma}^{2} \Delta t$ by $\Delta t$ and take the square root to produce the required $\hat{\sigma}$ for substitution into the Black Scholes formula.

\footnotetext{
${ }^{3}$ This notation for the Black Scholes formula suppresses the notation for its other variables, i.e. the riskless rate of return $r$, the time to expiration $T$, the current price of the underlying security, and the exercise price.

${ }^{4} \mathrm{~A}$ somewhat related contribution of Duan [10] used GARCH to model the actual underlying return process, assumed that the risk neutral conditional return distribution is still normal (albeit with altered mean), and assumed that its variance would equal the actual conditional variance. Härdle and Hafner [17] showed that the normal distribution with the smallest entropy relative to the actual normal distribution must have the same variance (albeit with altered mean).
} 


\subsection{Empirical Implementation}

To facilitate comparison with prior analyses, this procedure was applied using S \& P 500 returns between September 1985 and July 1994, the same data period used in Stutzer [28, p.1647]. Furthermore, the effects of dividends are built into these calculations, using the method described there.

The historical volatility over the entire period (including the Crash of 1987) was around $\sigma=$ $15 \%$, with small variations dependent on the $\Delta t$ used to estimate it. For options with 4 weeks to expiration, the $\hat{\sigma}-\sigma$ was about +2.5 percentage points . For options with 12 weeks to expiration, $\hat{\sigma}-\sigma$ rose to +3.5 percentage points. For options with longer time to expirations, $\hat{\sigma}-\sigma$ was lower, dropping back to around +2.5 percentage points for options with 24 weeks to expiration. If this reinterpretation of the Black-Scholes volatility parameter has merit, these upward adjustments should be close to the average difference over the estimation period of the option-implied volatilities $\sigma_{i m p}$ from the realized asset volatilities .

Empirical evidence in Fleming (op.cit.) is consistent with these predicted upward adjustments. Over the period October 1985 through April 1992, which overlaps with our period but excludes the Crash of 1987, Fleming compared the implied volatilities of nearest-to-the-money S \& P 100 index options that were closest to expiration (between 15 and 47 days), to their corresponding realized asset volatility to expiration. Fleming's Table 1 shows that the average upward bias of call option implied volatilities over realized asset volatilities was 1.9 percentage points, while that for put options was +2.5 percentage points. While Fleming's call option bias estimates are somewhat lower than the aforementioned $\hat{\sigma}-\sigma=+2.5$ percentage points predicted for options expiring in this range, Fleming's estimates would have been higher had they incorporated data surrounding the Crash of October 1987, as the predictions did. For as noted by Fleming (op.cit., p.7), "including these data actually increases the bias since the crash induced a subsequent longterm increase in implied volatility which outweighs the understatement of volatility on October 19." In summary, the only published quantitative evidence, the direction and magnitude of BlackScholes stock index option pricing errors during a lengthy historical period that includes the Crash of 1987 , is consistent with the entropic hypothesis. ${ }^{5}$

\section{Conclusions}

In the theory of contingent claims valuation with complete, frictionless markets, the price of a contingent claim written on underlying assets is its expected present value, using a riskless discount rate and an equivalent martingale measure to compute the expectation. In the best known case of a (call) option to buy a single underlying asset with IID normal return at a predetermined exercise

\footnotetext{
${ }^{5}$ Clearly, more extensive empirical testing should be the subject of another paper.
} 
price, relative entropy minimization provides a simple way to compute the martingale measure that yields the important Black-Scholes option pricing formula. The Black-Scholes formula depends on a parameter that is usually interpreted as the volatility of the underlying (normal) asset return process. But when returns are non-normal, the relative entropy calculation indicates an adjustment of this "volatility" parameter that helps capture the effects of non-normality. Existing empirical evidence for $S \& P 500$ stock index options is consistent with this generalization of the Black-Scholes model.

\section{References and Notes}

1. Amemiya, T. Advanced Econometrics; HarvardUniversity Press, 1985.

2. Avellanada, M. Minimum relative-entropy calibration of asset-pricing models. International Journal of Theoretical and Applied Finance 1998, 1, 447-472.

3. Bates, D.S. Post-'87 Crash fears in S\&P 500 futures options. Finance Dept., College of Business Administration, University of Iowa, Iowa City, IA 52242-1000, June 1997.

4. Ben-Tal, A. The entropy penalty approach to stochastic programming. Mathematics of Operational Research 1985, 10(2), 263-279.

5. Buchen, P.W.; Kelly, M. The maximum entropy distribution of an asset inferred from option prices. Journal of Financial and Quantitative Analysis 1996, 31, 143-159.

6. Bucklew, J.A. Large Deviation Techniques in Decision, Simulation, and Estimation; Wiley, 1990.

7. Cozzolino, J.M.; Zahner, M.J. The maximum entropy distribution of the future market price of a stock. Operations Research 1973.

8. Csiszar, I. I-divergence geometry of probability distributions and minimization problems. Annals of Probability 1975, 3(1), 146-158.

9. Dothan, M.U. Prices in Financial Markets; Oxford University Press, 1990.

10. Duan, J.-Ch. The GARCH option pricing model. Mathematical Finance 1995, 5(1), 13-32.

11. Fleming, J. The quality of market volatility forecasts implied by S\& P 100 index option prices. Jones Graduate School of Administration, Rice University, Houston, TX 77005, Feb. 17, 1997.

12. Follmer, H.; Schweizer, M. Hedging of contingent claims under incomplete information. In Applied Stochastic Analysis; Davis, M.H.A; Elliott, R.J., Eds.; Gordon and Breach, 1990.

13. Fosterand, F.D.; Whiteman, C.H. An application of Bayesian option pricing to the soybean market. American Journal of Agricultural Economics 1999, 81, 722-272.

14. Fritelli, M. The minimal entropy martingale measure and the valuation problem in incomplete markets. Mathematical Finance 2000, 10.

15. Gerber, H.; Shiu, E. Option pricing by Esscher Transforms. Transactions of the Society of Actuaries 1994, 46, 99-140.

16. Gulko, L. The entropy theory of option pricing. Working Paper, Department of Finance, Yale University, 1995.

17. Hardle, W.; Hafner, C. Discrete time option pricing with fexible volatility estimation. Institut für 
Statistik and Okonometrie, Humboldt Universität zu Berlin, Spandauer Str.1, D-10178 Berlin, Germany; June1997.

18. Harrison, M.; Kreps, D. Martingales and arbitrage in multiperiod securities markets. Journal of Economic Theory 1979, 20, 381-408.

19. Hawkins, R.J.; Rubinstein, M.; Daniell, G.J. Reconstruction of the probability density function implicit in option prices from incomplete and noisy data. In Maximum Entropy and Bayesian Methods; Hanson, K.; Silver, R., Eds.; Kluwer, 1996.

20. He, H. Convergence from discrete to continuous-time contingent claim prices. Review of Financial Studies 1990, 3(4), 523-546.

21. Heston, S.L. Option pricing with infitely divisible distributions. Working Paper, Goldman Sachs Asset Management, 1997.

22. Longstaff, F.A. Option pricing and the martingale restriction. The Review of Financial Studies 1995, 8(4), 1091-1124.

23. Massimb, M. Reconstruction of the risk-neutral distribution of stock prices. Investment Section, Harris Bank, Chicago, IL, December 30, 1992.

24. Samperi, D. Entropy and statistical model selection for asset pricing and risk management. Working Paper, Samperi Research, January 1999.

25. Stoll, H.; Whaley, R. Futures and Options; South-Western, 1993.

26. Stutzer, M. The statistical mechanics of asset prices. In Differential Equations, Dynamical Systems, and Control Science; Elworthy, K.D.; Everett, W.N.; Lee, E.B.; Eds.; Volume 152 of Lecture Notes in Pure and Applied Mathematics; Marcel Dekker, 1994: pp 321-342.

27. Stutzer, M. A Bayesian approach to diagnosis of asset pricing models. Journal of Econometrics 1995, 68, 367-397.

28. Stutzer, M. A simple nonparametric approach to derivative security valuation. Journal of Finance 1996, 51(4), 1633-1652.

29. Zou, J.; Derman, E. Strike adjusted spread: A new metric for estimating the value of equity options. Quantitative Strategies Research Note, Goldman, SachsandCo., July 1999.

(C) 2000 by the author. Reproduction is permitted for noncommercial purposes. 